\title{
Ubiquitin-Protein Ligase E3A
}

National Cancer Institute

\section{Source}

National Cancer Institute. Ubiquitin-Protein Ligase E3A. NCI Thesaurus. Code C75437.

Ubiquitin-protein ligase E3A (875 aa, 101 kDa) is encoded by the human UBE3A gene.

This protein plays a role in both protein ubiquitination and protein turnover. 